\title{
¿QUÉ TIENEN EN CUENTA LOS PROFESORES CUANDO SELECCIONAN EL CONTENIDO DE ENSEÑANZA? CAMBIOS Y DIFICULTADES TRAS UN PROGRAMA DE FORMACIÓN
}

\author{
SÁNCHEZ BLANCO, GASPAR y VALCÁRCEL PÉREZ, MARIA VICTORIA \\ Departamento de Didáctica de las Ciencias Experimentales. Universidad de Murcia \\ Campus de Espinardo. 30100 Murcia \\ E-mail: gsblanco@fcu.um.es - mvvalcarcel@fcu.um.es
}

\begin{abstract}
SUMMARY
The selection and sequencing of syllabus content is one of the main tasks the teacher has to approach in his/her new role as curriculum designer. The teaching proposals produced should be theoretically-driven so that specific procedures are suggested for the analysis of disciplinary content and pupils' characteristics. Knowledge regarding teachers' beliefs about these proposals, the difficulties involved in learning them and their scope of application is necessary in order to analyze how adequate they might be for inducing educational changes. In this paper, we present the initial teaching beliefs and teaching practices of a group of secondary school teachers who had previously received a training course in which a teaching proposal was presented. Also, some information is outlined concerning their assessment of the proposal as well as the learning and educational changes effected.
\end{abstract}

\section{INTRODUCCIÓN}

Un modelo curricular con un carácter procesual, abierto y flexible, como el adoptado por la educación primaria y secundaria en España, demanda una participación activa del profesor y amplía sus responsabilidades implicándole en tareas de diseño, desde presupuestos constructivistas, como marco propicio para comprender el aprendizaje de las ciencias y tomar decisiones sobre su enseñanza. Dejar la responsabilidad exclusiva de esta tarea a especialistas a través de los libros de texto llevará, por una parte, a una escasa contextualización del currículo, ya que sus propuestas se realizan para ámbitos educativos generalizados, y, por otra, lleva al peligro de que los profesores las utilicen de forma mecánica y acrítica sin llegar a comprender suficientemente los fundamentos de las mismas, a menudo no suficientemente explícitos en las propuestas que las editoriales realizan. Sin embargo, este nuevo rol de diseñador plantea un difícil reto al profesor de ciencias, que ha sido cuestionado por diferentes especialistas al considerar que los profesores no están preparados para abordarlo, pues su formación no ha sido dirigida hacia esas demandas, y han destacado que requiere un cambio radical en sus concepciones y práctica docente (Jiménez y Sanmartí, 1995; Gil, et al., 1998).

La delimitación y secuenciación del contenido de enseñanza es sin duda una tarea clave para abordar el diseño de la enseñanza. Nosotros pensamos que esta tarea debe ser guiada a través de unos fundamentos y procedimientos hacia el análisis del contenido disciplinar y de las dificultades de su aprendizaje en función de las características de los alumnos, lo que constituye dos tareas interdependientes, como reflexión previa para la toma de decisiones en torno a la concreción no sólo de los objetivos y contenidos educativos sino también de las estra-tegias didácticas y de evaluación necesarias para el 
desarrollo y evaluación de las unidades didácticas (Sánchez y Valcárcel, 1993). Resultados de la investigación sobre el proceso de planificación ponen de manifiesto que la atención del profesor se dirige principalmente hacia el contenido disciplinar, siendo su selección la decisión que con más frecuencia se toma en primer lugar (Clark y Peterson, 1986; Bellon et al., 1992; Brickhouse, 1993; Sánchez y Valcárcel, 1999). Junto a esto, también se ha apuntado el papel destacado, y a veces exclusivo, que los libros de texto y las guías del profesor tienen en la selección del contenido de enseñanza (Bellon et al., 1992) y el hecho de que los profesores, como expertos en una disciplina más que como docentes, a menudo convierten los contenidos disciplinares en contenidos curriculares sin establecer diferencias (Porlán y Martín, 1994).

Por otra parte, parece obvio que las ideas, experiencias, capacidades y actitudes de los alumnos deben condicionar las decisiones de los profesores en sus tareas docentes, planificación y trabajo en el aula, aunque la forma y el grado en que lo hagan dependerá de las concepciones y estilos docentes de cada profesor. Algunos trabajos sobre las concepciones o conductas docentes de los profesores (Hacker, 1984; Ballenilla, 1992; Freire y Chorao, 1992; Mellado, 1998; Porlán et al., 1998) han resaltado el predominio de concepciones transmisivas sobre la enseñanza y de concepciones del aprendizaje que sitúan al alumno como un receptor de conocimientos, por lo que prestan escasa o nula atención a las dificultades que tiene éste en el aprendizaje de las ciencias. Esta situación contrasta con las nuevas demandas que se hacen a los profesores desde una concepción constructivista del aprendizaje. En este sentido, diferentes autores han analizado las competencias que debe tener un profesor para desempeñar el nuevo rol y, desde los resultados de la investigación en la didáctica de las ciencias experimentales, han formulado lo que se denomina conocimiento deseable del profesor (Brincones et al., 1986; Del Carmen, 1990; Sáez, 1990; Gil, 1991, 1993; Furió, 1994; Porlán y Martín, 1994; Jiménez y Sanmartí, 1995; Porlán et al., 1996; Kennedy, 1998). Este conocimiento es coherente con un enfoque constructivista del aprendizaje de las ciencias, aunque pueda traducirse en modelos didácticos diversos. De las diferentes aportaciones destacamos, por su relación con la problemática que abordamos en este trabajo, el amplio consenso en torno a las siguientes ideas:

- El profesor debería conocer en profundidad la disciplina, es decir, sus objetivos, problemas, leyes y teorías, su historia, la práctica de su metodología científica, su epistemología y las interacciones ciencia-tecnologíasociedad asociadas a su construcción.

Esto ha motivado que sea importante introducir, en la formación del profesorado, aspectos que profundicen en el conocimiento de la materia a enseñar, como la reflexión epistemológica de las disciplinas (Hodson, 1988; Jiménez, 1995; Duschl, 1997) y estrategias para el análisis y selección de contenidos científicos que ofrezcan una visión adecuada de la ciencia y sean interesantes para los alumnos (Gil, 1993).
- El profesor debería adquirir conocimientos fundamentados sobre el aprendizaje de las ciencias; saber detectar, analizar e interpretar las concepciones de los alumnos para orientar su aprendizaje, con especial atención al análisis de los posibles obstáculos que impiden la construcción de las ideas científicas y al establecimiento de posibles itinerarios para superarlos. Conocer la psicología y la sociología del adolescente es necesario para comprender y favorecer su aprendizaje.

En relación con ello, compartimos con Coll (1986) que la capacidad cognitiva del alumno es un factor determinante de lo que es capaz de hacer y aprender en cualquier situación, así como que ésta depende tanto de los conocimientos previos que tenga el alumno sobre el contenido de enseñanza en cuestión (Ausubel, 1976; Novak y Gowin, 1988) como de que tenga las habilidades intelectivas que se requieren para su comprensión; es decir, que las demandas cognitivas de las tareas que se plantean al estudiante estén en consonancia con su capacidad para resolverlas, no ya por sí mismo sino en las condiciones más favorables de aprendizaje. Tales habilidades varían para cada individuo en función del estadio de desarrollo operatorio en que se encuentra (Piaget e Inhelder, 1972; Shayer y Adey, 1984) y son dependientes del contenido concreto en que las han de aplicar (Coll, 1986; Pozo et al., 1991). Junto a lo anterior, también los propósitos e intereses de los alumnos han sido destacados por su importancia en lo que se aprende ante un programa de trabajo (Driver, 1988). En definitiva, el conocimiento de los alumnos y sus características es destacado como una categoría clave en la estructura de conocimiento que debe poseer un profesor.

- El profesor debería: a) tener criterios para la selección y secuenciación del contenido de enseñanza; $b$ ) saber formular, en relación con los objetivos y contenidos de enseñanza, una serie de metaconocimientos, un conjunto de procedimientos generales y una serie de valores básicos que sirvan de referente continuo para el proceso de enseñanza- aprendizaje; y $c$ ) en concreto, elaborar tramas de contenidos que relacionen la información procedente de las disciplinas científicas y problemas relevantes e interesantes para los alumnos.

Aunque compartimos con otros autores (Gil, 1993; Del Carmen, 1996) que la formalización de un procedimiento no va a reducir la complejidad del proceso de planificación, pensamos que el profesor, para construir su enseñanza, debe disponer de propuestas de criterios y estrategias que dirijan y fundamenten sus reflexiones y toma de decisiones.

Ahora bien, desde diferentes propuestas de organización del conocimiento profesional (Mellado,1996; Porlán et al., 1996, 1997), se destaca la importancia de los saberes derivados de la práctica de la enseñanza de los profesores, como una fuente de ese conocimiento. Este hecho nos debe hacer considerar que los conocimientos académicos, como los de la didáctica de las ciencias, son necesarios pero no suficientes para generar un nuevo conocimiento profesional deseable, ya que se yuxtaponen e integran con los derivados de su práctica. Además, 
si atendemos al proceso de aprendizaje que se requiere para la adquisición de cualquier conocimiento, no debe olvidarse que las concepciones de los profesores influyen decisivamente en sus aprendizajes y, por consiguiente, en los posibles cambios que se puedan generar en sus concepciones y prácticas como consecuencia de procesos formativos.

Coherentemente con todo lo anterior, sus concepciones y prácticas deberán considerarse punto de partida y eje de la formación (Hewson y Hewson, 1987; Porlán y García, 1992; Gil, 1993). Diversos investigadores han señalado también esta demanda por otros motivos, destacando que, si los cambios no son introducidos de manera que tomen en consideración las preocupaciones, intereses, creencias e ideas de los profesores respecto a los diferentes elementos que inciden en la enseñanza y aprendizaje de las ciencias, y respecto a cómo el cambio puede afectarles personalmente, es probable que su implementación no pueda ser nunca exitosa ni sustantiva (Fullan, 1991; Abell y Pizzini, 1992; Briscoe y Peters, 1997; Sánchez y Valcárcel, 1998).

Por otra parte, los programas que tienen como base el diseño y desarrollo del currículo y la investigación de la práctica docente han puesto de manifiesto que son una vía para promover el contraste y la integración de los diferentes saberes y fuentes del conocimiento profesional (Álvarez, 1988; Azcárate et al., 1994; Pedretti y Hodson, 1995; Martín et al., 1997; Parke y Coble, 1997; Gil, et al., 1998; Marx et al., 1998). Estos programas se muestran eficaces para iniciar los cambios didácticos que se pretenden, pues implican a los profesores en procesos de reflexión sobre su docencia, promueven prácticas colaborativas entre profesores y formadores, y desarrollan actitudes positivas y compromisos hacia la innovación. También muestran que la viabilidad de las innovaciones que se pretenden está condicionada por la disposición y formación previa de los participantes, y porque se adopte una perspectiva del cambio como un proceso a largo plazo que requiere que se proporcionen y mantengan apoyos durante su implementación.

Esta perspectiva de formación a largo plazo requiere analizar cuál es el perfil de uso de los profesores y cómo evoluciona éste ante propuestas formativas concretas, es decir, requiere identificar cuáles son los niveles de progresión que se producen, para fundamentar el diseño de posibles itinerarios formativos hacia el perfil de innovación deseable y tratar de establecer posibles obstáculos para el cambio que se pretende. Niveles de progresión no sólo desde la perspectiva de los aprendizajes que se puedan generar sino también, y no menos importante, desde la perspectiva de su valoración de los conocimientos y su actitud ante las nuevas demandas que se establecen.

Dentro de este marco, el objetivo de nuestro trabajo es el análisis de las concepciones y prácticas de un grupo de profesores en relación con la selección y secuenciación del contenido de enseñanza y en qué medida evolucionan tras un programa de formación. Con ello pretendemos establecer niveles de progresión y delimitar dificul- tades para el cambio, hacia un perfil de innovación coherente con el rol del profesor como diseñador del currículo. En concreto, en este artículo aportamos resultados y reflexiones en torno a tres cuestiones:

a) Qué tienen en cuenta los profesores cuando seleccionan y secuencian el contenido de enseñanza?

b) ¿Cómo valoran los profesores el contenido ofertado en un curso de formación desde determinados criterios necesarios para que sea utilizado en su práctica docente (comprensión, validez formativa, compatibilidad, viabilidad e interés)?

c) ¿Cuáles son los aprendizajes y cambios docentes generados en relación con la propuesta formativa y las principales dificultades encontradas?

\section{METODOLOGÍA DE LA INVESTIGACIÓN}

\section{Descripción de la muestra}

Los sujetos con los que se ha realizado el estudio han sido 27 profesores de educación secundaria de los cuales 13 eran profesores diplomados en ciencias, 8 licenciados en química y 6 licenciados en biología, todos con más de dos años de experiencia y la mayoría con más de diez. También era la primera vez que todos asistían a un curso de actualización científica didáctica dirigido a profesores de ciencias experimentales.

\section{Instrumentos de recogida de información}

Para la recogida de información se utilizaron diferentes instrumentos, tratando de que respondieran tanto a intereses formativos como de la investigación. Así, con las entrevistas iniciales se buscaba dar respuesta al primero de los interrogantes señalados. Para ello, antes del curso, pedimos a todos los profesores que contaran qué hacen cuando planifican su curso y preparan sus unidades didácticas, lecciones o temas. Al finalizar el curso de formación, de nuevo fueron entrevistados para pedirles explicaciones sobre los contenidos seleccionados y los criterios utilizados en el diseño de la unidad didáctica que realizaron durante el curso. Las entrevistas, de tipo estructurado, fueron analizadas básicamente siguiendo el procedimiento descrito por Miles y Huberman (1984), quienes diferencian tres actividades: $a$ ) la reducción de datos, que nos permitió establecer las categorías; $b$ ) la exposición de datos, en nuestro caso mediante matrices, para organizar el conjunto de la información; c) la extracción de conclusiones, para el establecimiento de regularidades y explicaciones.

Otro instrumento utilizado fue un cuestionario cerrado de 25 ítems que describen las ideas claves de los contenidos del programa de formación (fundamentos y procedimientos). Los ítems fueron valorados por los profesores, en una escala de 1 a 5 , desde cinco criterios, cuyos significados hicimos explícitos en el cuestionario. Tales criterios son: comprensión (el grado en que el profesor 
ha podido entender la información), validez formativa (el grado en que la información aportada la considera útil en su formación), compatibilidad (el grado en que puede asumir o aceptar el contenido tratado desde sus actuales ideas, creencias...), viabilidad (el grado en que piensa que el contenido aportado puede ser transferible a su práctica docente) e interés (el grado en que el contenido le ha resultado atractivo, motivador...). Como hemos mostrado en otros trabajos (Sánchez, 1997; Sánchez y Valcárcel, 1998), la selección de estos criterios no es arbitraria sino que responde a cualidades que se exigen a cualquier conocimiento que se oferta a los profesores para garantizar su uso en los centros escolares. Esta información constituye la dimensión subjetiva del conocimiento que necesitan los profesores para que sean posibles los cambios que se prentenden con la formación. Por tanto, con la información del cuestionario pretendemos dar respuesta al segundo de los interrogantes planteados.

Por último, las unidades didácticas diseñadas por los profesores han sido el instrumento utilizado, junto con la entrevista final, para dar respuesta al tercer interrogante planteado. Los profesores, en grupos de dos o tres, diseñaron un unidad didáctica tras la primera fase del curso sobre un tópico elegido libremente por ellos. En total se diseñaron doce unidades sobre temáticas diversas: dinámica; calor y temperatura; energía; inmunología; alteraciones de los ecosistemas; fotosíntesis; el agua; el aire; etc. En éstas se recogen por escrito el análisis del contenido de enseñanza, las posibles dificultades de aprendizaje y las implicaciones didácticas que, consecuentemente, han tenido en cuenta. Es decir, con los documentos escritos podemos conocer sus decisiones sobre la selección y secuenciación del contenido de enseñanza.

El análisis de las unidades didácticas, lo hemos realizado desde tres criterios (seguimiento, coherencia y realización), buscando una correspondencia con diferentes niveles de aprendizaje propuestos por Joyce y Showers (1988): sensibilización, comprensión y aprehensión. El último nivel que proponen estos autores, la transferencia, no ha sido considerado, pues habría requerido que los profesores diseñaran autónomamente una unidad didáctica en el contexto de sus centros escolares, lo que quedaba fuera del objetivo del curso de formación.

Desde el criterio de seguimiento, hemos valorado en qué medida los profesores siguen el procedimiento que proponemos para la selección y secuenciación del contenido; con el de coherencia, en qué medida las decisiones de los profesores responden al significado dado en la propuesta; y, por último, con la realización, en qué medida se alcanzan los objetivos planteados en estas tareas incluidas en el diseño de la unidad didáctica. Para cada uno de estos criterios hemos categorizado los aprendizajes de los profesores con la valoración de óptimo, aceptable y no aceptable.

Finalmente señalamos que la reflexión conjunta o triangulación de los datos obtenidos con los instrumentos señalados (entrevistas, cuestionario y documentos escri- tos) y con los registros del diario de sesiones de los formadores y de las grabaciones audiovisuales nos ha permitido profundizar en nuestro análisis, incrementando la fiabilidad y validez del estudio, y sintetizar nuestros hallazgos, los cuales son discutidos en la siguiente sección.

\section{Programa de formación}

Dado que asumimos que el conocimiento que se oferta a los profesores está determinado tanto por el contenido como por la estrategia de formación, nos referiremos brevemente a ambos aspectos del programa para situar con mayor claridad la propuesta que hacemos para la selección y secuenciación del contenido de enseñanza. En otros trabajos (Sánchez, 1997; Sánchez y Valcárcel, 1998) se recoge con mayor detalle el programa de formación desarrollado.

Los contenidos del programa, que son objeto de análisis en el presente trabajo, forman parte básicamente del desarrollo de las dos primeras tareas del modelo de planificación que hemos propuesto para el diseño de unidades didácticas (Sánchez y Valcárcel, 1993). Éstos incluyen tanto unos fundamentos para la toma de decisiones del profesor sobre la selección y secuenciación del contenido como unos procedimientos concretos para llevar a cabo esta tarea desde criterios disciplinares (análisis científico) y criterios derivados de la consideración de los alumnos (análisis didáctico). De modo sintético se recogen en el cuadro I los contenidos de estas dos tareas. Durante el curso, el tiempo total presencial que se dedicó a estos contenidos fue de 36 horas.

La estrategia de formación se diseñó siguiendo las recomendaciones que diferentes autores (Yeany y Padilla, 1986; Showers et al., 1987; Marcelo y Estebaranz,1991) dan para que la modalidad de cursos de formación sea más eficaz. Así, contemplamos tres fases con objetivos diferenciados: instruir a los profesores sobre el conocimiento deseable, dar oportunidades a los profesores para que utilicen esa información de manera práctica y valorar la incidencia del conocimiento en la práctica docente de los profesores.

\section{RESULTADOS DE LA INVESTIGACIÓN}

Los resultados se presentan en tres secciones en correspondencia con los interrogantes planteados. En la primera, se analizan las concepciones y práctica iniciales de los profesores en relación con la selección y secuenciación del contenido de enseñanza, ya que caracterizar la situación inicial nos permite establecer el nivel de partida para poder analizar su evolución y determinar los aspectos cuyo cambio resulta más o menos problemático. En la segunda, se presenta cómo valoran los profesores la nueva propuesta, pues una valoración positiva es requisito para que se inicien los procesos de cambio y, por el contrario, una valoración negativa puede explicar sus dificultades o la falta de adecuación a su práctica docente. En la tercera, se analiza qué han aprendido los 
profesores y cuáles son las principales dificultades para incorporar la nueva propuesta a su práctica docente.

La descripción de los resultados cualitativos es acompañada con frecuencia del porcentaje de profesores que están incluidos en las categorías diferenciadas, lo que permite aproximarnos a un perfil global de la muestra estudiada por encima de las diferencias individuales.

\section{Concepciones y prácticas iniciales de los profesores}

En una publicación anterior hemos realizado una descripción amplia acerca de las concepciones y prácticas de los profesores cuando realizan la planificación corta de su enseñanza (Sánchez y Valcárcel, 1999). En aquélla analizamos el procedimiento que siguen para preparar sus unidades didácticas, temas o lecciones, los elementos que tienen en cuenta y el valor y la utilidad que le otorgan a su planificación. En este trabajo, retomaremos los resultados acerca de cómo seleccionan y secuencian el contenido de enseñanza, ampliándolos con datos sobre la práctica de la planificacion larga o anual, para profundizar en cuál es el papel relativo que juegan los contenidos disciplinares y los alumnos en su toma de decisiones.

\section{El elemento clave de la planificación es el contenido disciplinar}

Cuando realizan la programación anual del curso, los criterios mayoritarios que utilizan los profesores para definir sus unidades de programación son el contenido disciplinar $(70 \%)$ y el tiempo $(81 \%)$, en general de modo dependiente. Los profesores procuran que las unidades tengan coherencia incluyendo contenidos científicamente relacionados, pero condicionando su extensión por el tiempo disponible para todo el programa. Es frecuente encontrar distribuciones como: bloque temático por trimestre o mes, tema por semana y lección por sesión. Este papel central del contenido disciplinar tambien se refleja al preparar sus unidades, temas o lecciones, ya que todos los profesores reconocen que comienzan planteándose el contenido de la unidad, como es patente en el siguiente comentario:

Profesor 10: «Considerando el tiempo que voy a dedicar a esa lección, selecciono los contenidos que se van a estudiar y, dentro de ellos, los aspectos concretos que más me interesa que aprendan, ya que los considero más importantes. Dentro de cada pregunta hay alguna cosas superfluas y otras importantes. Lo esencial es que se queden con unas cuantas ideas claras, lo que podríamos llamar ideas clave de cada pregunta o de cada lección.»

Aunque algunos de ellos, todos diplomados en ciencias, expresan tener como referencia los objetivos recogidos en la programación anual $(22 \%)$, en realidad se refieren a los contenidos mínimos que deben aprender los alumnos. De hecho, la mayoría del profesorado (64\%) manifiesta que el contenido de la disciplina es el elemento más importante cuando preparan sus lecciones, dando explicaciones como la siguiente:
Profesor 5: «Creo que [lo más importante es] establecer los contenidos que tienen que asimilar, que tiene que saber el alumno al final del curso. Lo demás no son más que herramientas para conseguir eso; o sea, que el alumno alcance al final unos objetivos mínimos sobre la asignatura.»

Por último señalaremos que, en cualquiera de las situaciones anteriores, los profesores se limitan a hacer una lista para secuenciar los contenidos seleccionados, pero no analizan ni definen los esquemas conceptuales que están implícitos en los mismos, lo que consideramos necesario para fundamentar su secuenciación.

\section{El libro de texto es una referencia básica para la plani-} ficación

Sus principales referencias para realizar la programación anual son las planificaciones de años anteriores $(52 \%)$, los programas oficiales $(48 \%)$ o los libros de texto $(48 \%)$. A estos últimos se refieren como algo oficial que recoge los contenidos del curso y justifican su selección, o las modificaciones que realizan, desde su experiencia, como ejemplifica la siguiente opinión:

Profesor 23: «Viene todo estructurado en la guía del profesor. Estudias el libro y sacas todos los objetivos, los contenidos que tienen que saber, los que te parecen más razonables, más llevaderos y más importantes.»

De nuevo, para la selección y secuenciación del contenido de las unidades, casi todos utilizan como referencia básica el libro de texto del alumno o del profesor. Para unos $(33 \%)$, es la guía exclusiva y, para otros (59\%), un apoyo fundamental, como refleja la siguiente opinión:

Profesor 8: «No puedo ponerme a explicar y que luego los alumnos no tengan en el libro lo que estoy explicando. Si hay un tema que no está como yo quiero, porque le falta, pues, entonces, les dicto lo que falta y, si sobra algo, por estar muy elevado o no me gusta, les digo que lo quiten. Luego siempre me gusta darles colecciones de problemas de otros libros para que tengan más material.»

Como en este caso, al seleccionar el contenido a través del libro de texto, a veces los profesores $(59 \%)$ realizan modificaciones en función de la importancia que le conceden y el tiempo disponible; e incluso, para algunos (25\%) también los alumnos, sus intereses o nivel en general, condicionan estas modificaciones. En general, el mayor o menor compromiso con el libro de texto está condicionado por el nivel educativo o curso, tomándose como guía en los niveles más bajos y como apoyo para los superiores, sobre todo en bachillerato o en COU.

Las modificaciones al libro de texto, en la mayoría de los casos, se refieren a una reducción de contenidos, a su simplificación o al cambio de una definición por otra que les parece más adecuada, pero no suponen cambios sustanciales a los esquemas conceptuales que se plantean o subyacen en el texto ni propuestas alternativas a los mismos. Además, en ningún caso mencionan criterios científicos o didácticos objetivos para explicar su 
selección del contenido o las modificaciones que introducen en el libro de texto, pues sólo aluden a su experiencia. Nos referimos a criterios coherentes con los contenidos del programa de formación, es decir, que deriven de sus conocimientos sobre la naturaleza de las disciplinas o sobre las capacidades cognitivas de los alumnos que se requieren para el aprendizaje de los contenidos seleccionados.

\section{El contenido de enseñanza es exclusivamente teórico}

En cuanto a la naturaleza de los contenidos de enseñan$\mathrm{za}$, todos los profesores se plantean, casi con exclusividad, contenidos teóricos, lo que es coherente con el carácter básicamente conceptual de los libros de textos. Así, los profesores, cuando hablan del contenido, se refieren al desarrollo de la teoría y más de la mitad no aluden a actividades experimentales. El carácter conceptual de la enseñanza queda refrendado por la ocasional o nula realización de actividades experimentales, pues, como explica un profesor, el laboratorio no es rentable:

Profesor 4: «Las prácticas de laboratorio... hace mucho tiempo que no las hago. Para que una experiencia de laboratorio sea rentable, el alumno tiene que tener los conocimientos teóricos suficientes para que le saque una aplicación. En química, los alumnos no tienen ni idea y, para entretenerlos, no los bajo al laboratorio, por supuesto. Además, tengo la experiencia de un temario muy amplio y al final me falta tiempo.»

Por otra parte, quienes realizan actividades experimentales $(41 \%)$ suelen hacerlo como complemento del contenido teórico desarrollado, lo que lleva a que se planifiquen sólo ocasionalmente. Tan sólo en dos casos el trabajo experimental es un contenido de enseñanza relevante para el profesor al ser importante para el logro de sus objetivos.

\section{Los profesores tienen en cuenta el nivel o conocimientos generales de sus alumnos}

Aunque los profesores hacen espontáneamente escasas referencias a los alumnos (11\%) y, en su caso, lo hacen de forma genérica aludiendo a sus intereses o nivel como criterio para seleccionar el contenido de enseñanza, cuando se les pregunta en la entrevista expresamente por si consideran a los alumnos al preparar sus clases y por el papel que éstos juegan, todos admiten tenerlos en cuenta en un momento u otro del proceso; incluso el $52 \%$, entonces, manifesta que su procedimiento comienza considerando los conocimientos de los alumnos. Sin embargo, los comentarios de los profesores, al ser preguntados expresamente, son ilustrativos de cómo los consideran:

Profesor 14: «Sí los tengo en cuenta porque te limitan totalmente. Una cosa es que pongas sobre el papel una programación y otra, que luego te viene cada personaje... que te limita totalmente. Hay 30 críos [...] con muchas deficiencias en lectura, conocimientos ...en cuanto a todo, eso te condiciona muchísimo. Aunque haya selec- cionado los contenidos previamente a partir de la idea de otros años, siempre intento adaptar los contenidos al tipo de alumnos que llegan. Hay cosas que... me doy cuenta, que serán una pérdida de tiempo y que no les va a servir para nada; entonces, pues, intento adaptarlo al tipo de personas que vienen.»

Las precisiones que hacen los profesores, sobre lo que saben los alumnos, según lo hayan dado en cursos anteriores o según se trate de cursos buenos o malos, parece indicar que, cuando preparan sus lecciones, están condicionados de algún modo por ellos, pues parten de cuánto saben para decidir qué deben saber. Sin embargo, esta consideración queda distante de una reflexión substancial sobre las posibilidades de aprendizaje que tienen de los contenidos concretos de la unidad, lo que explica que no mencionen espontáneamente a los alumnos cuando describen el procedimiento que siguen para preparar sus unidades. La poca trascendencia que tiene el alumno en la selección de los contenidos concretos de una unidad también se puso de manifiesto cuando les pedimos que destacaran el elemento más importante para la toma de decisiones, pues sólo tres profesores (11\%) resaltan el nivel, conocimientos e intereses de los alumnos.

Por tanto, aunque los profesores dicen que tienen en cuenta los conocimientos de los alumnos sobre la materia, de sus respuestas puede inferirse que se refieren más al conocimiento genérico que tienen de la disciplina que al contenido concreto de cada unidad. También con carácter general, unos pocos profesores $(27 \%)$ se refieren a la capacidad intelectual de los alumnos, a su comprensión lectora y a su capacidad de expresión, pero en ningún caso a capacidades relacionadas con esquemas de conocimiento específicos necesarios para la comprensión de los contenidos de ciencias (Shayer y Adey, 1984). Podemos concluir, por consiguiente, señalando que las ideas previas y la adecuación de los contenidos en función de las exigencias cognitivas concretas que requiere su comprensión no son referentes para el profesorado cuando consideran al alumno.

La experiencia docente es la fuente del conocimiento sobre el alumno

Para diagnosticar el nivel de los alumnos o sus conocimientos generales, los profesores recurren a dos vías: la realización de una prueba escrita al inicio del curso, cuando todavía no conocen a los alumnos, y la propia experiencia, vía ésta que consideran más que suficiente sobre todo cuando los han tenido otros años. Aunque la mitad del profesorado no se refiera a ningún procedimiento, parece lógico pensar, por comentarios como el que sigue, que la vía de la experiencia es la forma natural de tener en cuenta el nivel, los conocimientos y la capacidad intelectual de los alumnos:

Profesor 27: «Yo sé los alumnos que tengo. He estado 18 años trabajando en un colegio y sé el nivel que hay y el tipo de alumnos que tengo. Sé que hay cursos muy graciosos que cogen las cosas muy bien y otros que no.» 
Este conocimiento, fruto de la experiencia docente, lleva a la mayoría a realizar modificaciones que se centran en una adecuación general de la programación anual (67\%) con la intención de simplificar la materia prevista, de incidir más en algunos contenidos o de fijar objetivos mínimos. En otros casos (33\%), las modificaciones inciden menos en la selección del contenido, pues se centran más en adaptar las explicaciones al nivel del alumno.

A la vista de los resultados anteriores, podemos concluir que el hacer de los profesores durante la selección y secuenciación de los contenidos de enseñanza está muy alejado de la propuesta que se les plantea. Además, dado que la mayoría $(66 \%)$ valora positivamente el resultado de su trabajo, aunque asumen que es posible mejorar, y pocos se refieren a la necesidad de conocer mejor la materia a enseñar (11\%) o conocer mejor a los alumnos $(11 \%)$, podemos concluir que existe una situación inicial no deseable desde un enfoque constructivista del aprendizaje, dada la poca insatisfación inicial de los profesores con su práctica docente y la escasa demanda de formación relativa a los contenidos del programa que se les oferta. Ante esta situación, a menudo frecuente en una fase de iniciación de la formacion permanente o, en general, cuando los profesores acceden a ella por otros motivos diferentes a los formativos -como, por ejemplo, el requisito administrativo para obtener un sexenio-, se hace necesario prestar especial atención a las actividades de formación, que tienen por objeto el planteamiento de situaciones problemáticas y de conflicto para que los profesores reflexionen y cuestionen sus concepciones y prácticas actuales.

\section{Dimensión subjetiva del contenido de formación}

Durante el desarrollo del curso de formación, en diferentes momentos, los profesores manifiestaron abiertamente su valoración, en general positiva, tanto de los contenidos desarrollados como de los diferentes tipos de actividades realizadas (exposiciones, trabajos en grupo, puestas en común) y de los documentos escritos aportados. Aunque no es objeto de este trabajo valorar estos resultados, los señalamos por la incidencia obvia que pueden tener en la valoración del contenido de formación. En cualquier caso, de los resultados se desprende que, para los profesores, el interés de los contenidos formativos está condicionado principalmente por las cualidades que les asignan a los mismos y, secundariamente, por el desarrollo de la estrategia de formación.

\section{Valoración del contenido del análisis científico (AC)}

En relación con los contenidos relativos al análisis científico (Cuadro I), los resultados muestran que los profesores declaran tener una opinión excelente de su comprensión, pues más del $80 \%$ considera que ha sido buena o muy buena, aunque los formadores, por el seguimiento de sus trabajos, destacaron la existencia de dificultades para la comprensión del criterio de selección del contenido disciplinar y la propuesta de análisis de los contenidos procedimentales.

La validez formativa aparece siempre con una valoración altamente positiva (más del $75 \%$ ), destacando nuevamente la propuesta para la selección del contenido y la inclusión de los procedimientos como contenidos de enseñanza. Así mismo, destaca por el alto grado de compatibilidad la propuesta en su conjunto y, sobre todo, los criterios que definen la unidad didáctica y los mapas de conceptos como procedimiento adecuado para la estructuración del contenido, lo que ya habían argumentado en diferentes momentos del curso porque les había permitido clarificar sus ideas sobre el contenido de enseñanza.

También destaca la mayor viabilidad práctica de los mapas de conceptos y el criterio para definir la unidad didáctica, frente a una menor viabilidad del procedi-

Cuadro I

Contenidos del programa de formación para el análisis del contenido de enseñanza

\begin{tabular}{|l|l|}
\hline \multicolumn{1}{|c|}{ Análisis científico (AC) } & \multicolumn{1}{c|}{ Análisis didáctico (AD) } \\
\hline$>$ Importancia de las creencias científicas de los profesores. & $>\begin{array}{l}\text { Condicionantes generales para el proceso de enseñanza- } \\
\text { aprendizaje. }\end{array}$ \\
$>$ Concepciones de la ciencia e implicaciones didácticas. & $>$ Conocimientos previos de los alumnos y aprendizaje escolar. \\
$>\begin{array}{l}\text { Clasificación del conocimiento científico y tipos } \\
\text { de contenidos de enseñanza. }\end{array}$ & $>$ Investigaciones sobre ideas de los alumnos en ciencias. \\
$>\begin{array}{l}\text { Criterios para la selección y secuenciación del contenido } \\
\text { (identificación, interpretación y aplicación). }\end{array}$ & $>$ Exigencias cognitivas de los contenidos. \\
$>$ Los mapas de conceptos como instrumentos para el AC. & $>$ Esquemas necesarios para la comprensión de las ciencias. \\
$>\begin{array}{l}\text { Estrategia para la selección de contenidos procedimentales: } \\
\text { el método de preguntas de Gowin. }\end{array}$ & $>\begin{array}{l}\text { Utilización de las tareas razonadas y taxonomías de Shayer y } \\
\text { Adey para el análisis del currículo. }\end{array}$ \\
\hline $\begin{array}{l}\text { Clasificación de contenidos actitudinales. } \\
\text { Criterios de selección. }\end{array}$ & $>$ Implicaciones para la planificación de la enseñanza. \\
\hline
\end{tabular}


miento propuesto para la selección de los contenidos procedimentales, basado en la V de Gowin (Novak y Gowin, 1988). Además, durante el desarrollo de este contenido, se planteó una amplia discusión sobre las posibilidades reales de que pudieran trabajarse en el aula los contenidos procedimentales, cuestionando su viabilidad en las circunstancias actuales con argumentos como la extensión de los programas, el tiempo requerido, los medios disponibles, etc., sobre todo para los niveles superiores de la eduación secundaria.

Por último, se deduce que el interés de la mayor parte del contenido ha sido muy positivo para más del $75 \%$ de los profesores, a excepción de los referidos a las concepciones sobre la naturaleza de la ciencia y la técnica de análisis de los procedimientos. Probablemente, querer fundamentar la estrategia de reflexión para el análisis del contenido desde el proceso de construcción del conocimiento científico ha podido ser un objetivo ambicioso, para el tiempo disponible, dadas las diferencias con el perfil inicial del profesorado. Por ello, estos contenidos pueden haber sido percibidos por los profesores como algo teórico y explicar su menor motivación.

\section{Valoración del contenido del análisis didáctico $(A D)$}

En relación con los contenidos desarrollados sobre las posibilidades de aprendizaje de los alumnos (Cuadro I), los resultados obtenidos muestran, desde todos los criterios, una diferencia considerable entre la valoración que los profesores hacen de aquéllos relacionados con las ideas de los alumnos y los relativos a las exigencias cognitivas de los contenidos. Conviene señalar que en ningún caso las valoraciones consideradas negativas $(1,2)$ son asignadas por más del $25 \%$ de los sujetos, en el caso menos favorable, y en la mayoría de los casos lo son por un porcentaje sensiblemente menor. Por tanto, las diferencias que presentamos deben entenderse siempre dentro de una tendencia positiva en la valoración del contenido desarrollado.

Así, considerando las valoraciones más positivas (puntuaciones 4 y 5), la comprensión de los aspectos relativos a las ideas de los alumnos es considerada buena o muy buena por más del $77 \%$ de los sujetos, en el peor de los casos, mientras que la valoración de los relacionados con las exigencias cognitivas de los contenidos disminuye claramente, hasta el punto de que sólo el $25 \%$ dicen haber comprendido bastante o mucho el procedimiento propuesto para su análisis. De los primeros, nos parecen destacables las características de las ideas de los alumnos, su utilidad para explicar dificultades del aprendizaje y la necesidad de tenerlas en cuenta en la acción docente para favorecer el aprendizaje de las ciencias, por haber sido valorados con las máximas puntuaciones por más del $80 \%$ de los profesores desde todos los criterios. Además, durante el curso y tras el desarrollo de estos contenidos, algunos profesores ya manifestaron espontáneamente que compartían la importancia de las ideas de los alumnos y la necesidad de considerarlas.

Como consecuencia, reconocieron que se mostraban más sensibles en sus clases porque conocían las concep- ciones de sus estudiantes y habían introducido alguna actividad específica para diagnosticarlas. Esta excelente valoración y evolución producida puede explicarse, en parte, porque se trata de un tema cercano a las observaciones cotidianas de los profesores en el aula y que pueden constatar con cierta facilidad.

La resultados de la comprensión sobre las exigencias cognitivas son coherentes con las dificultades señaladas por los profesores, durante el desarrollo del curso, al tratar de dar respuesta a los trabajos planteados. Los profesores asumen que los alumnos de cada nivel tienen diferentes capacidades y, por tanto, que los contenidos pueden ser más o menos complejos en función de que dispongan de las habilidades intelectivas necesarias para su comprensión. Sin embargo, las dificultades surgen cuando los profesores deben analizar si un contenido concreto es adecuado, o no, en función del nivel de los alumnos. El procedimiento que propusimos para llevar a cabo esta tarea fue considerado por los profesores de forma menos positiva, aumentando el porcentaje de quienes valoraron como regular (puntuación 3) su comprensión, validez formativa y, consiguientemente, la compatibilidad con sus concepciones y su viabilidad práctica.

Estos resultados creemos que se pueden explicar, en parte, por el tiempo dedicado a ello y por la propia complejidad del procedimiento propuesto, lo cual no favoreció su comprensión. Además, durante el trabajo práctico, se puso de manifiesto que los profesores tenían dificultades para identificar los esquemas de conocimiento implícitos en los contenidos de enseñanza, requisito necesario para analizar las exigencias cognitivas de esos contenidos. Las taxonomías de Shayer y Adey (1984) fueron presentadas como un instrumento útil para analizar el nivel de exigencia de los contenidos en función de los estadios operatorios de los alumnos. Se trataba de una aproximación cualitativa que podía servir para explicar o prever determinadas dificultades de aprendizaje y, en consecuencia, tomar decisiones durante la planificación. Probablemente, los profesores esperaban que les proporcionáramos una forma más operativa y sencilla de diagnosticar el nivel de los alumnos y la adecuación de los contenidos seleccionados.

En conclusión, aun existiendo las dificultades mencionadas, la importancia del análisis del contenido desde la perspectiva disciplinar y desde los potenciales aprendizajes de los alumnos, como reflexión previa para delimitar y fundamentar la selección y secuenciación del contenido de enseñanza y la concreción de los objetivos de las unidades didácticas, es valorada positivamente por la mayoría del profesorado desde las diferentes cualidades consideradas. Estos resultados nos llevan a pensar que la propuesta formativa es adecuada para iniciar a los profesores en los cambios que se demandan.

\section{Aprendizajes y cambios docentes}

Los resultados obtenidos muestran la conveniencia de diferenciar niveles de aprendizaje para apreciar los logros formativos y la necesidad de valorar los mismos 
desde una perspectiva de los cambios docentes que generan o potencialmente pueden generar.

Valoración de los aprendizajes desde el perfil de innovación

La utilización de la propuesta formativa para la selección y secuenciación del contenido de enseñanza requería llevar a cabo el conjunto de acciones siguiente:

a) Analizar y seleccionar el contenido desde el criterio disciplinar planteado (identificación, interpretación y aplicación).

b) Definir el esquema conceptual de la unidad.

c) Delimitar, con la estrategia planteada (método de preguntas de Gowin), los procedimientos científicos que pueden ser contenidos de enseñanza.

d) Señalar dificultades de aprendizaje fundamentadas desde las ideas previas de los alumnos y desde las exigencias cognitivas de los contenidos.

e) Delimitar implicaciones para la enseñanza de los contenidos seleccionados, tanto en relación con su secuencia como con las estrategias didácticas.

f) Seleccionar los objetivos de enseñanza desde la consideración conjunta de los resultados de los análisis científico y didáctico.
En la tabla I se recoge en qué medida los profesores llevan a cabo el conjunto de acciones señaladas teniendo como referencia los tres criterios adoptados: seguimiento, coherencia y realización. Para facilitar la lectura del análisis de los resultados, que realizamos a continuación, iremos identificando los diferentes aspectos a los que nos referimos con la letra $(a, b, c, d, e, f)$ asignada en la tabla.

Los resultados muestran que todos los profesores analizan y seleccionan los contenidos $a$ y definen el esquema conceptual de la unidad didáctica $b$ siguiendo la propuesta realizada, haciéndolo la mayoría en el grado óptimo. Sin embargo, esta situación desciende al $25 \%$ cuando han tenido que delimitar los contenidos procedimentales $c$, siendo considerable el porcentaje (41\%) que no llegan a realizarlo. Los resultados, respecto a las dos primeras tareas, siguen siendo positivos para los criterios de coherencia y realización; sin embargo, la valoración óptima no es mayor del $50 \%$ en ningun caso, siendo para la mayoría de los trabajos sólo aceptable. Cabe resaltar que, aunque utilicen el criterio de identificación, interpretación y aplicación para seleccionar el contenido, en muchos casos su empleo no fue adecuado por una falta de comprensión del mismo, como reconocieron los profesores, aunque a su vez consideraron que había sido útil para reflexionar sobre los contenidos de enseñanza que debían seleccionar. Esta insuficiente comprensión puede deberse al hecho de que no siempre resulta evidente diferenciar, en torno a un contenido científico, el nivel descriptivo y el nivel explicativo, lo que corres-

Tabla I

Resultados del aprendizaje de la propuesta desde los criterios seleccionados.

\begin{tabular}{|l|lll|}
\hline & Seguimiento & Coherencia & Realización \\
\hline a) Selección de contenidos & $\begin{array}{l}\text { óptimo (75\%) } \\
\text { aceptable (25\%) }\end{array}$ & $\begin{array}{l}\text { óptimo (41\%) } \\
\text { aceptable (41\%) } \\
\text { no aceptable (16\%) }\end{array}$ & $\begin{array}{l}\text { óptimo (25\%) } \\
\text { aceptable (58\%) } \\
\text { no aceptable (16\%) }\end{array}$ \\
\hline b) Esquema conceptual & $\begin{array}{l}\text { óptimo (83\%) } \\
\text { aceptable (16\%) }\end{array}$ & $\begin{array}{l}\text { óptimo (33\%) } \\
\text { aceptable (41\%) } \\
\text { no aceptable (25\%) }\end{array}$ & $\begin{array}{l}\text { óptimo (25\%) } \\
\text { aceptable (50\%) } \\
\text { no aceptable (25\%) }\end{array}$ \\
\hline c) Procedimientos científicos & $\begin{array}{l}\text { óptimo (25\%) } \\
\text { no reabliza (41\%) }\end{array}$ & $\begin{array}{l}\text { óptimo (33\%) } \\
\text { aceptable (8\%) } \\
\text { no aceptable (16\%) }\end{array}$ & $\begin{array}{l}\text { óptimo (33\%) } \\
\text { no aceptable (25\%) }\end{array}$ \\
\hline d) Dificultades de aprendizaje & $\begin{array}{l}\text { óptimo (41\%) } \\
\text { aceptable (58\%) }\end{array}$ & $\begin{array}{l}\text { óptimo (58\%) } \\
\text { aceptable (25\%) } \\
\text { no aceptable (16\%) }\end{array}$ & $\begin{array}{l}\text { óptimo (41\%) } \\
\text { aceptable (41\%) } \\
\text { no aceptable (16\%) }\end{array}$ \\
\hline e) Implicaciones para la enseñanza & $\begin{array}{l}\text { óptimo (41\%) } \\
\text { no realiza (58\%) }\end{array}$ & $\begin{array}{l}\text { óptimo (33\%) } \\
\text { aceptable (8\%) }\end{array}$ & $\begin{array}{l}\text { óptimo (25\%) } \\
\text { aceptable (16\%) }\end{array}$ \\
\hline aceptable (66\%) Selección de objetivos & $\begin{array}{l}\text { óptimo (16\%) } \\
\text { aceptable (75\%) } \\
\text { no aceptable (8\%) }\end{array}$ & $\begin{array}{l}\text { óptimo (16\%) } \\
\text { aceptable (50\%) } \\
\text { no aceptable (33\%) }\end{array}$ \\
\hline
\end{tabular}


ponde con la identificación e interpretación respectivamente, porque los conceptos que describen los hechos tienen su origen en las teorías científicas que explican los mismos y su enseñanza puede tener un enfoque descriptivo o interpretativo, que estará supeditado al fenómeno que estemos estudiando y, por consiguiente, a los diferentes niveles de complejidad con los que puede abordarse.

En relación con el análisis procedimental $c$, sólo la tercera parte lo realiza de forma coherente con el análisis conceptual anterior en términos suficientemente precisos. Los profesores explicaron esta situación refiriéndose, en algún caso, a que aún se mostraban confusos sobre el concepto de contenido procedimental y, en otros, a la complejidad que para ellos tenía el análisis utilizando estrategias como el método de preguntas de Gowin.

Todos los grupos analizaron cuáles eran las dificultades para el aprendizaje $d$, haciéndolo la mayoría en términos más que aceptables, pues sólo se alcanza un porcentaje negativo del $16 \%$ desde los criterios de coherencia y realización. Los buenos resultados obtenidos, a pesar de la problemática señalada acerca de las exigencias cognitivas en el apartado anterior, están justificados porque decidimos valorar conjuntamente la identificación de dificultades para el aprendizaje, con independencia de que se analizaran las ideas previas o las exigencias cognitivas. Como cabía esperar, mientras que todos los profesores contemplan en su planificación las ideas previas de los alumnos y, además, la mayoría lo hace de modo coherente con la propuesta formativa, sólo el $41 \%$ consideró las exigencias cognitivas de los contenidos y tan sólo el $16 \%$ las analizó siguiendo la propuesta que presentamos. La mayor parte de los profesores justificaron no haber analizado las exigencias cognitivas de los contenidos por haberles resultado complejo el procedimiento propuesto. El desarrollo cognitivo de los alumnos es sin duda un área de interés para los profesores, pero es necesario facilitarles el análisis de las exigencias cognitivas del contenido a través de instrumentos y propuestas que les permitan diagnosticar los niveles de los alumnos y estudiar las posibles adecuaciones del contenido de una forma más operativa para ellos. Creemos que sería necesario profundizar en propuestas como las de Shayer y Adey o buscar otras alternativas para dar soluciones a este problema haciendo más accesible este conocimiento al profesorado de ciencias.

Los resultados del siguiente procedimiento $e$ muestran porcentajes menos satisfactorios, pues el 58\% no explicitó implicaciones para la enseñanza. Aquellos grupos $(41 \%)$ que abordaron esta parte lo hicieron en términos óptimos o aceptables tanto desde el punto de vista de coherencia como de realización, pues señalaron implicaciones para la secuenciación del contenido, la selección de objetivos o actividades en términos suficientemente concretos. Durante las entrevistas finales, algunos profesores justificaron no haber derivado implicaciones para la enseñanza, una vez identificadas las dificultades de aprendizaje que tienen los alumnos, por no haber comprendido suficientemente la propuesta. La falta de tiempo y su implicación insuficiente en el trabajo a realizar fueron también motivos dados por los profesores para justificar las diferencias entre los diseños realizados y la propuesta que se les había planteado.

En cuanto a la selección de objetivos $f$, existe una elevada correspondencia entre las decisiones de los profesores al precisar sus intenciones educativas y los resultados de los análisis precedentes, en la mayoría de la muestra. Sin embargo, todavía algunos no consideran de forma explícita los resultados del análisis didáctico, o al menos no lo hacen coherentemente, lo que nos lleva a pensar que el planteamiento de objetivos para estos profesores sigue teniendo como principal referencia el contenido disciplinar.

Del conjunto de resultados anteriores, podemos concluir que, si bien los profesores se han mostrado sensibles por utilizar la propuesta procedimental, en su conjunto, dados los porcentajes considerados aceptables u óptimos en el seguimiento de la misma, lo hacen con un nivel de comprensión desigual que resulta insuficiente, en algunos casos, para que puedan incorparla autónomamente a su práctica docente, dados los porcentajes valorados como aceptables u óptimos en los niveles de coherencia y realización. Los resultados globales, que sintetizan los aprendizajes realizados desde los tres criterios anteriores, ratifican esta conclusión y muestran diferentes niveles de progresión entre los asistentes. Concretamente hemos diferenciado a los profesores en tres grupos en función de los resultados óptimos o aceptables en los tres criterios:

Grupo 1. Formado por ocho profesores que realizaron las tres unidades didácticas que han sido valoradas como excelentes. En más del $80 \%$ de los aspectos considerados $(a, b, c, d, e \mathrm{y} f)$, los resultados son óptimos.

Grupo 2. Formado por ocho profesores que realizaron las cuatro unidades didácticas que han sido bien valoradas. Entre el $50 \%$ y el $80 \%$ de los aspectos anteriores obtienen un resultado óptimo o aceptable.

Grupo 3. Formado por once profesores que realizaron las cinco unidades didácticas que no son bien valoradas.Los resultados óptimo o aceptable que consiguen no alcanzan el $50 \%$ de los aspectos considerados.

Esto muestra que los objetivos que nos planteamos se han alcanzado en diferente medida en cada caso. Así, el profesorado del primer grupo ha mostrado una capacitación para el análisis del contenido de enseñanza muy satisfactoria, de la que cabría esperar cambios significativos en su práctica docente en la planificación y en el aula. Aunque en menor medida, el profesorado del segundo grupo también ha mostrado una capacitación suficiente para pensar que podría plantearse algunas de las innovaciones que se pretendían con la actividad de formación. Por último, el del tercer grupo, aunque haya diseñado las unidades didácticas incorporando algunas referencias de la propuesta planteada, nos parece que no se encuentra preparado suficientemente para acometer innovacio- 
nes importantes en relación con el perfil de innovación que se perseguía.

\section{Valoración de la progresión del perfil inicial de los profesores}

Ahora bien, al considerar la evolución que se produce desde el perfil inicial de los profesores, creemos que lo aprendido ha supuesto logros importantes para casi todos los profesores. Así, con el contenido de enseñanza, los profesores han pasado de asumir el delimitado en el libro de texto a reflexionar sobre la problemática científica del mismo, lo que ha supuesto que mayoritariamente diferencien y seleccionen contenidos de naturaleza conceptual, procedimental y actitudinal; en grado alto, incorporan y diferencian contenidos de identificación, interpretación y aplicación; también, analizan e identifican el esquema conceptual que plantea la unidad didáctica, elaborando mapas de conceptos, e incluyen procedimientos científicos como contenidos propios de la enseñanza.

De igual modo, aunque el alumno era una preocupación de los profesores que caracterizaba su perfil de uso en la preparación de sus lecciones, no se planteaban la importancia de sus ideas previas relativas al contenido de enseñanza para la selección y secuenciación del contenido. Este logro es, por tanto, una novedad destacable. Sin embargo, aunque la sensibilización de los profesores por la importancia de las ideas previas nos parece que es un hecho necesario, pensamos que se debe incidir más en las repercusiones que deben derivarse en los planteamientos metodológicos, para evitar en lo posible que se conviertan en una anécdota del proceso de enseñanzaaprendizaje.

Por último, creemos que la mayoría, al diseñar la unidad didáctica muestra una concepción de los objetivos diferente a la expresada en el perfil inicial, pues, cuando los profesores se planteaban su necesidad, se limitaban a reformular en términos operativos los contenidos mínimos de enseñanza. Ahora, por los resultados, pensamos que el carácter reflexivo de las tareas precedentes, la problemática científica del contenido y las dificultades de aprendizaje de los alumnos se ponen de manifiesto por buena parte de los profesores, tanto al delimitar los contenidos adecuados al nivel educativo como al concretar los objetivos de las unidades.

\section{Cambios docentes generados}

En relación con los cambios docentes que se han podido generar en los profesores y reconociendo las limitaciones que tiene pretender indagar en los cambios que promueve un programa de formación sin contar con la observación directa del aula, deseábamos conocer la valoración que los propios sujetos tenían de los cambios ya realizados y cuáles eran sus expectativas sobre los mismos a corto plazo. Por ello, durante la entrevista preguntamos a los profesores en este sentido.

Prácticamente todos los profesores admiten que la actividad de formación ha cuestionado sus ideas sobre los contenidos de enseñanza, la amplitud de los programas y su carácter casi exclusivamente conceptual, y les ha llevado a relativizar su importancia frente a los aprendizajes de los alumnos, como explica el siguiente profesor:

UD2: «Claro, me ha hecho dudar de lo que estoy haciendo. Yo no sé si estoy dando demasiados contenidos y si verdaderamente hay que dar tantos porque los críos al final no aprenden... A lo mejor lo aprenden pero de una manera mecánica, te hacen los ejercicios en clase, te responden, pero realmente no lo han entendido... Eso si me hizo reflexionar y, desde luego, pienso que tengo que modificar la manera de enseñar.»

Parece lógico que, desde un enfoque transmisivo, el elemento clave del proceso de enseñanza-aprendizaje sea el contenido científico, del mismo modo que desde un enfoque constructivista lo será el alumno que construye este conocimiento. Por tanto, este cambio de enfoque que apreciamos entre los profesores en el análisis del proceso de enseñanza-aprendizaje puede deberse a que se admiten como plausibles los principios constructivistas del aprendizaje científico, lo que nos parece un cambio cualitativo importante en las ideas de los profesores. De hecho, la mitad de los entrevistados afirmaron haber introducido ya algunos cambios en su aula, en un esfuerzo por aumentar la participación de los alumnos en las actividades y manifestaron su percepción positiva del desarrollo de éstas. También indicaron haber utilizado los mapas de conceptos para la estructuración del contenido y haber realizado actividades para el diagnóstico de las ideas previas de los alumnos, considerándolas en sus explicaciones y actividades de enseñanza. El resto justificó no haber introducido todavía modificaciones en su práctica por el carácter cerrado de sus programaciones o por no haber tenido un nivel de comprensión suficiente de la propuesta hasta el final de la actividad de formación.

\section{Dificultades para los cambios que se demandan}

En relación con sus previsiones de incorporar las innovaciones en el curso siguiente, la mayoría admite la posibilidad pero resaltando que el proceso de cambio es necesario que ocurra paulatinamente, sobre todo por la dedicación que requiere el diseño de todas las unidades didácticas desde estos planteamientos. Una mayoría de los profesores $(85 \%)$ considera que implicarse en el diseño de unidades didácticas es en sí mismo un problema por exigirles una mayor dedicación, más trabajo y más tiempo del que disponen o están dispuestos a emplear, como muestra el siguiente comentario:

UD1: «Sí muy difícil; tiene sus dificultades... de tiempo. Hay que dedicar más tiempo y más tiempo, el profesor... El trabajo previo es muy incómodo, muy extenso y lleva mucho trabajo.»

Con independencia de los motivos por los cuales los profesores justifican la dificultad de poder aumentar su dedicación laboral en tareas de planificación, nos parece que este problema es determinante en los procesos de 
cambio y dudamos que sea suficientemente considerado por las instituciones educativas. Resulta obvio resaltar que cualquier modificación que conlleve una mejora educativa requiere una dedicación laboral mayor de los profesores. No se deben promover cambios docentes institucionales sin adoptar medidas que incidan en un mayor reconocimiento profesional y que incentiven la mayor dedicación que requiere.

Otro problema relacionado con esto, que señalan los profesores (44\%), es la falta de coordinación y de trabajo en equipo entre las personas que componen la unidad organizativa más inmediata, el seminario o ciclo. Con opiniones como la que sigue, destacan que los cambios sólo serán posibles si superan el ámbito individual, al exigirles a los mismos un carácter social, hecho que debería hacer reflexionar a los responsables de las instituciones que realizan las ofertas formativas, pues en general sólo se dirigen a personas, buscando la mejora de su competencia docente y la promoción personal, lo que es necesario pero no suficiente:

UD8: «A mí (Profesor 1), lo que me gustaría es que el resto de la gente de ciencias que hay en mi centro, o en el que sea, conociera, por lo menos, el sistema y, aunque todas las unidades no las vamos a poder programar en este sentido, ir introduciendo ya poco a poco esta nueva estructuración. Lo que pasa es que los cambios son lentos.»

Profesor 2: «En mi centro piensan que esto es mío. Y esto es para el centro... Se han interesado poquísimo. Cuando venía de la sesión, no te preguntaban "¿Qué haces?" No es un tema individual y mientras los demás compañeros no participen...»

En la problemática que nos ocupa, esta coordinación que demandan y compartimos es esencial en el proceso de cambio por diferentes motivos. Por un lado, porque el diseño y desarrollo del currículo de un centro debe ser una tarea compartida por todos los implicados en él y no la suma o yuxtaposición de proyectos individuales para cada curso, según el profesor que los imparte. Por otro lado, porque enriquecería y daría seguridad a los profesores en sus decisiones sobre la selección de los contenidos, su adecuación al alumno y a los objetivos didácticos, como consecuencia de la reflexión conjunta. Y por último, porque la mayor coordinación haría posible una distribución del trabajo que paliaría en parte el problema de la mayor dedicación que require implicarse en las tareas de planificación.

Por último, nos referiremos a un problema derivado de la opinión que los profesores pueden tener sobre la demanda del nuevo rol de diseñador. Algunos profesores, todos pertenecientes al tercer grupo caracterizado cuando comentamos los resultados globales de aprendizaje, no consideran justificada la necesidad de ser diseñadores de su enseñanza, pues opinan que disponen de excelentes libros de texto elaborados por expertos. Para ellos, aunque puedan admitir entre sus expectativas la posibilidad de introducir algunas innovaciones, los cambios que realicen serán anecdóticos, al menos en cuanto a su implicación en el proceso de planificación, ya que el libro de texto seguirá siendo la guía casi exclusiva de su enseñanza. En estos casos, la confianza de los profesores en las propuestas elaboradas por otros supone un obstáculo importante para que evolucionen hacia el perfil de diseñadores del currículo.

\section{CONCLUSIONES}

La selección y secuenciación del contenido de enseñanza es una tarea necesaria en el nuevo rol del profesor como diseñador de unidades didácticas, que es demandado por el carácter abierto de los actuales currículos de educación primaria y secundaria. Los resultados sobre las concepciones y prácticas de los profesores, cuando planifican su enseñanza, muestran un perfil de actuación que se caracteriza básicamente porque: $a$ ) el contenido disciplinar es el elemento clave del proceso; $b$ ) el libro de texto constituye la referencia fundamental para su selección y secuenciación del contenido; $c$ ) el contenido de enseñanza tiene un carácter exclusivamente teórico; d) el conocimiento que el profesor tiene del alumno incide escasamente en su toma de decisiones durante el proceso de planificación; e) las concepciones de los alumnos sobre el contenido concreto de las unidades didácticas no lo consideran. En definitiva, el hacer de los profesores se encuentra alejado de presupuestos coherentes con concepciones actuales sobre la ciencia y el aprendizaje científico.

Los fundamentos y procedimientos propuestos en un curso de formación sobre la selección y secuenciación del contenido de enseñanza en el diseño de unidades didácticas han sido valorados por los profesores, en general, en términos positivos desde diferentes criterios que configuran su dimensión subjetiva, concretamente por su comprensión, validez formativa, compatibilidad con sus ideas, viabilidad en la práctica e interés de la propuesta, por lo que cabría esperar que los aprendizajes de los profesores fueran igualmente positivos, así como su disposición a incorporar las innovaciones a su práctica docente. Sin embargo, los resultados encontrados ponen de manifiesto las diferencias en los logros formativos, los aprendizajes y los cambios docentes generados, en relación tanto con el contenido ofertado como con los sujetos que reciben el conocimiento.

En relación con el contenido ofrecido, las dificultades se centran fundamentalmente en dos puntos, que constituyen obstáculos importantes para evolucionar hacia el perfil de innovación:

a) El primero, que el análisis y selección de los contenidos procedimentales no se hace contemplando que éstos deben ser al mismo tiempo estrategias para el aprendizaje de los contenidos conceptuales y el desarrollo cognitivo de los alumnos (Sanmartí et al., 1990; Sánchez y Valcárcel, 1993), por lo que se traducen, en general, en simples habilidades de investigación más o menos manipulativas. Esta dificultad puede explicarse por la escasa 
implicación del profesorado en procesos de reflexión sobre el conocimiento científico y sus estrategias de construcción, bien sea durante su formación científica inicial o durante su desarrollo profesional, por lo que instrumentos como el método de preguntas de Gowin es considerado complejo y no se ve justificado su uso ante el esfuerzo y la dedicación temporal que requiere. Además, la incorporación de los contenidos procedimentales como contenidos de enseñanza es cuestionada, a menudo, por razones como la extensión de los programas, la falta de recursos, el número de alumnos por aula, etc., problemas que van más allá del conocimiento y actitudes del profesor ante estos contenidos.

b) La segunda dificultad encontrada es el análisis de las exigencias cognitivas de los contenidos, pues identificar los esquemas de conocimiento que están implícitos en el aprendizaje de los contenidos ha sido una tarea que consideran difícil y, más aún, determinar si el nivel de desarrollo que tienen los alumnos es adecuado a los mismos.

Para ello, creemos que es necesario elaborar materiales que sean de fácil aplicación en sus aulas y que proporcionen información concreta sobre esta problemática. Desde la investigación educativa debería profundizarse en propuestas, como la de Shayer y Adey, que aplicadas a nuestro contexto educativo aporten soluciones más accesibles al profesorado en este ámbito. Si bien enseñar a los profesores a elaborar esos materiales sería, en principio, un objetivo en su formación, ponerlos a su disposición ya elaborados facilitaría el acceso del profesor a ese conocimiento y reduciría las diferencias entre su perfil de uso y el de innovación que se pretende. Con esto reconocemos que existen diferentes niveles de implicación y autonomía de los profesores en el proceso de planificación de su enseñanza.

Sin embargo, otros contenidos de la propuesta que hacemos, como la utilización del criterio de identificación, interpretación y aplicación en la selección del contenido disciplinar y el uso de los mapas de conceptos, en la estructuración del contenido seleccionado, tienen menos dificultad en ser incorporados progresivamente a su

\section{REFERENCIAS BIBLIOGRÁFICAS}

ABELL, S.K. y PIZZINI, E.L. (1992). The effect of a problem solving in-service program on the classroom behaviors and attitudes of middle school science teachers. Journal of Research in Science Teaching, 29(7), pp. 649-667. práctica docente. De igual modo, los profesores no manifiestan dificultades para incorporar en su práctica las implicaciones que se derivan de considerar la importancia de las ideas previas de los alumnos en el aprendizaje y, por consiguiente, aceptan el papel determinante que deben tener para la selección y secuenciación del contenido.

Coherentemente con lo anterior, los profesores asumen que la selección y secuenciación del contenido de enseñanza deben fundamentarse y decidirse teniendo en cuenta conjuntamente el contenido disciplinar y el alumno y que su resultado debe reflejarse en la concreción de los objetivos didácticos. Así, los resultados muestran que, en general, todos fueron sensibles al uso de la propuesta que hacemos, lo que parece indicar una aceptación de los objetivos de la misma. Sin embargo, existen diferencias notables en cuanto al grado en que la han comprendido y pueden incorporarla de forma coherente a su práctica. Por ello hemos identificado tres niveles de progresión diferentes: $a$ ) en el superior se encuentran quienes podrían incorporar los criterios y procedimientos propuestos en el diseño de sus unidades de forma autónoma; $b$ ) en el intermedio, los que todavía necesitarían el apoyo de asesores para clarificar la propuesta e incorporarla a su práctica; y $c$ ) en el inferior, los que están aún lejos de poder implicarse en tareas de diseño curricular con los objetivos que se pretendían.

Por último, un problema común a todos los profesores, que debe superarse para implicarlos en el diseño del currículo, es la necesidad de tener que dedicar un mayor tiempo a las tareas docentes, lo que no ha sido contemplado por la administración educativa cuando ha planteado un currículo abierto y pide la participación de los docentes en su concreción. La falta de incentivos para esta mayor dedicación se puede traducir en buscar soluciones para resolver esta tarea en el menor tiempo posible, por ejemplo, asumiendo las propuestas que diferentes editoriales realizan a través de los libros de texto, lo que en definitiva lleva nuevamente a una práctica docente en la que el profesor sigue siendo un mero ejecutor de un currículo cerrado.
ÁLVAREZ, R.M. (1988). Una experiencia de asesoramiento a profesores/as: dinamización del seminario de ciencias naturales de un centro de bachillerato. Investigación en la Escuela, 6 , pp. 81-86. 
AUSUBEL, D.P. (1976). Psicología educativa: Un punto de vista cognoscitivo. México: Trillas.

AZCÁRATE, P., CUESTA, J., NAVARRETE, A. y CARDEÑOSO, J.M. (1994). Presupuestos iniciales para un trabajo de investigación sobre formación del profesorado. Investigación en la Escuela, 22, pp. 85-89.

BALLENILLA, F. (1992). El cambio de modelo didáctico, un proceso complejo. Investigación en la Escuela, 18, pp. 43-54.

BELLON, J.J., BELLON, E.C. y BLANK, M.A. (1992).Teaching from a research knowledge base: A development and renewal process. Nueva York: Macmillan Publishing Company.

BRICKHOUSE, N.W. (1993). What counts as successful instruction? An account of a teacher's self-assessment. Science Education, 77, pp. 115-129.

BRINCONES, I., FUENTES, A., NIEDA, J., PALACIOS, M.J. y OTERO, J. (1986). Identificación de comportamientos deseables del profesorado de ciencias experimentales del bachillerato. Enseñanza de las Ciencias, 4(3), pp. 209-222.

BRISCOE, C. y PETERS, J. (1997). Teacher collaboration across and within schools: Supporting individual change in elementary science teaching. Science Education, 81(1), pp. 51-65.

CLARK, C.M. y PETERSON, P.L. (1986). Procesos de pensamiento de los docentes, en Wittrock, M.C. La investigación de la enseñanza III: Profesores y alumnos, pp. 443-539. Barcelona: Paidós.

COLL, C. (1986). Bases psicológicas. Cuadernos de Pedagogía, 139, pp. 12-16.

DEL CARMEN, L. (1990). Desarrollo curricular y formación permanente del profesorado, en Gil, D. (ed.). Formación de formadores en didáctica de las ciencias, pp. 45-58. Valencia: Nau LLibres.

DEL CARMEN, L. (1996). Análisis y secuenciación de los contenidos educativos. Barcelona: Universitat de Barcelona, ICE- Horsori.

DRIVER, R. (1988). Un enfoque constructivista para el desarrollo del currículo de ciencias. Enseñanza de las Ciencias, 6 (2), pp. 109-120.

DUSCHL, R. (1997). Renovar la enseñanza de las ciencias. Importancia de las teorías y su desarrollo. Madrid: Narcea.

FREIRE, A.M. y CHORAO, M.F. (1992). Elements for a typology of teachers' conceptions of physics teaching.Teaching and Teacher Education, 8 (5/6), pp. 497-507.

FULLAN, M. (1991). The new meaning of educational change. Londres: Cassel Education.

FURIÓ, C. (1994). Tendencias actuales en la formación del profesorado de ciencias. Enseñanza de las Ciencias, 12 (2), pp. 188-199.

GIL, A., GONZÁLEZ, E., MIYAR,C. y ALDABA, J. (1998). Asesoramiento y formación del profesorado de ciencias de bachillerato LOGSE. Análisis de una experiencia en el País Vasco. Alambique, 15, pp. 29-37.

GIL, D. (1991). ¿Qué hemos de saber y saber hacer los profesores de ciencias? (Intento de síntesis de las aportaciones de la investigación didáctica). Enseñanza de las Ciencias, 9 (1), pp. 69-77.

GIL, D. (1993). Aportaciones de la didáctica de las ciencias a la formación del profesorado, en Montero, L. y Vez, J.M. (eds.). Las didácticas específicas en la formación del profesorado I, pp. 277-293. Santiago de Compostela: Torculo.

GIL, D., FURIÓ, C. y GAVIDIA, V. (1998). El profesorado y la reforma educativa en España.Investigación en la Escuela, 36, pp. 49-64.

HACKER, R.G. (1984). A typology of approaches to science teaching in schools. European Journal of Science Education, 6(2), pp. 153-167.

HEWSON, P.W. y HEWSON, M.G.(1987). Science teachers' conception of teaching: Implications for teacher education. International Journal of Science Education, 9, pp. 425-440.

HODSON, D. (1988). Towards a philosophically more valid science curriculum. Science Education, 72, pp. 19-40.

JIMÉNEZ, M.P. (1995). Comparando teorías: La reflexión sobre la naturaleza de la ciencia en la fomación del profesorado, en Blanco, L.J. y Mellado, V. (coord.). La formación del profesorado de ciencias y matemáticas en España y Portugal, pp. 267-280. Badajoz: Diputación Provincial.

JIMÉNEZ, M.P. y SANMARTÍ, N. (1995). The development of a new science curriculum for secondary school in Spain: opportunities for change. International Journal of Science Education, 17(4), pp. 425-439.

JOYCE, B. y SHOWERS, B. (1988). Student achievement through staff development. Nueva York: Longman

KENNEDY, M. (1998). Education reform and subject matter knowledge. Journal of Research in Science Teaching, 35(3), pp. 249-263.

MARCELO, C. y ESTEBARANZ, A. (1991). Estrategias de formación centradas en el contenido y en el formador (estrategias deductivas), en Escudero, J.M. y López, J. (coord.). Los desafios de las reformas escolares. Cambio educativo y formación para el cambio, pp. 179-218. Sevilla: Arquetipo Ediciones.

MARTÍN, J., CANTARERO, M.A. y NÚÑEZ, P. (1997). Una experiencia de formación como punto de partida de un cambio metodológico. Investigación en la Escuela, 31, pp. 99-111.

MARX, R., FREEMAN, J.,KRAJCIK, J. y BLUMENFELD, P. (1998). Professional development of science teachers, en Fraser, B.J. y Tobin, K.G. (eds.). International Handbook of Science Education II, pp. 667-680. Dordrecht: Kluwer Academic Publishers.

MELLADO, V. (1996). Concepciones y prácticas de aula de profesores de ciencias, en formación inicial, de primaria y secundaria. Enseñanza de la Ciencias, 14(3), pp. 398-302.

MELLADO, V. (1998). La investigación sobre el profesorado de ciencias experimentales, en Banet, E. y De Pro, A. (coords.) Investigación e innovación en la enseñanza de las ciencias I, pp. 272-283. Murcia: DM.

MILES, M. y HUBERMAN, A. (1984). Qualitative data analysis. Beverly Hills: Sage.

NOVAK, J.D. y GOWIN, D.B. (1988). Aprendiendo a aprender. Barcelona: Martínez Roca.

PARKE, H.E. y COBLE, C.R. (1997). Teachers designing curriculum as professional development: A model for transformational science teaching. Journal of Research in Science Teaching, 34(8), pp. 773-789.

PEDRETTI, E. y HODSON, D. (1995). From rhetoric to action: Implementing STS education through action research. Journal of Research in Science Teaching, 32(5), pp. 463-485. 
PIAGET, J. y INHELDER, B. (1972). De la lógica del niño a la lógica del adolescente. Buenos Aires: Paidós.

PORLÁN, R., AZCÁRATE, P., MARTÍN, R., MARTÍN, J. y RIVERO, A. (1996). Conocimiento profesional deseable y profesores innovadores: fundamentos y principios formativos. Investigación en la Escuela, 29, pp. 23-38.

PORLÁN, R. y GARCÍA, M.V. (1992). The change of teachers' conceptions: A strategy for in-service science teachers' education. Teaching and Teacher Education, 8(5/6), pp. 537-548.

PORLÁN, R. y MARTÍN, J. (1994). El saber práctico de los profesores especialistas. Aportaciones desde las didácticas específicas. Investigación en la Escuela, 24, pp. 49-58.

PORLÁN, R., RIVERO, A. y MARTÍN, R. (1997). Conocimiento profesional y epistemología de los profesores I: teoría, métodos e instrumentos. Enseñanza de las Ciencias, 15(2), pp. 155-171.

PORLÁN, R., RIVERO, A. y MARTÍN, R. (1998). Conocimiento profesional y epistemología de los profesores II: estudios empíricos y conclusiones. Enseñanza de las Ciencias, 16(2), pp. 271-288.

POZO, J.I., GÓMEZ CRESPO, A.M., LIMÓN, M. y SANZ, A. (1991). Procesos cognitivos en la comprensión de la ciencia: Las ideas de los adolescentes sobre la química. Madrid: CIDE-MEC.

SÁEZ, M.J. (1990). El reto de un cambio insoslayable. La formación del profesorado de ciencias. Enseñanza de las Ciencias, 8(2), pp. 144-152
SÁNCHEZ, G. (1997). Diseño, desarrollo y evaluación de un programa de formación, sobre la planificación de unidades didácticas, para el profesorado de ciencias en ejercicio de educación secundaria. Tesis doctoral. Universidad de Murcia.

SÁNCHEZ, G. y VACÁRCEL, M.V. (1993).Diseño de unidades didácticas en el área de ciencias experimentales. Enseñanza de las Ciencias, 11(1), pp. 33-44.

SÁNCHEZ, G. y VALCÁRCEL, M.V. (1998). La planificación de unidades didácticas en la formación permanente del profesorado de ciencias: dimensión subjetiva del contenido de formación, en Banet, E. y De Pro, A. (coords.).Investigación e innovación en la enseñanza de las ciencias I, pp. 324-335. Murcia: DM.

SÁNCHEZ, G. y VALCÁRCEL, M.V. (1999). Science teachers' views and practices in planning for teaching. Journal of Research in Science Teaching, 36(4), pp. 493-513.

SANMARTÍ, N., MAURI, T., IZQUIERDO, M. y GÓMEZ, I. (1990). Los procedimientos. Área ciencias de la naturaleza. Cuadernos de Pedagogía, 180, pp. 28-32.

SHAYER, M. y ADEY, P. (1984). La ciencia de enseñar ciencias. Madrid: Narcea.

SHOWERS, B., JOYCE, B. y BENNETT, B. (1987). Synthesis of research on staff development: A framework for future study and a state-of-the-art analysis. Educational Leadership, 45(3), pp. 77-87.

YEANY, R.H. y PADILLA, M.J. (1986). Training science teachers to utilize better teaching strategies: A research synthesis. Journal of Research in Science Teaching, 23(2), pp. 85-95.

[Artículo recibido en septiembre de 1999 y aceptado en febrero de 2000.] 